Espacio, Tiempo y Forma, S. III, H. ${ }^{a}$ Medieval, t. 4, 1991, págs. 79.94

\title{
La Corona de Castilla en el siglo XV. La Administración Central
}

\author{
Vicente Ángel Álvarez Palenzuela *
}

\section{LA MONARQUIA}

Como en la práctica totalidad del Occidente europeo, durante toda la Edad Media, la forma de gobierno en Castilla, en el siglo XV, es la Monarquía. El Rey es la encarnación suprema del Reino y la doctrina monárquica se halla sólidamente fundamentada en principios cristianos - desde San Agustín a Santo Tomás- que hacen al monarca reflejo de la unidad divina, a la que representa; la unidad es superior a la pluralidad, según el pensamiento medieval, de modo que el orden reside en el poder de uno, del mismo modo que el poder plural conduce a la anarquía.

De esta forma, el régimen monárquico nunca fue puesto en teia de juicio, aunque sí se discutiera, y de forma muy áspera, el modo de ejercicio de la prerrogativa regia. Dos corrientes se enfrentan en esta interpretación: el autoritarismo y el pactismo.

El autoritarismo se apoya en principios de Derecho Romano y reclama para la Monarquía la plenitud de la potestad regia; cobra importancia debido a la notable difusión del Derecho Romano y a los legistas que colaboran con los monarcas y que están imbuidos de estas ideas.

El pactismo parte de la idea de que toda autoridad procede de la comunidad, y su ejercicio no consiste sino en la aplicación de la ley, que

- Catedrático de H. ${ }^{a}$ Medieval. Universidad Autónoma de Madrid. 
es el resultado de la costumbre consolidada en el tiempo, y del respeto a los privilegios individuales y colectivos de los súbditos. Existiría un contrato tácito entre el monarca y sus súbditos según el cual éstos se comprometen a obedecer las disposiciones de aquél, que, a su vez, está obligado a respetar el ordenamiento jurídico del Reino. La consecuencia evidente es que los súbditos están legitimados para resistir aquellos actos de gobierno que, por ser contrarios a la ley y la costumbre, son, en realidad, actos tiránicos.

Los dos principios teóricos constituyen el eje de gran parte de la política castellana, y peninsular en general, durante la Baja Edad Media, en especial durante el siglo XV. El autoritarismo proporcionó la base teórica para reclamar para el Rey el "poder real absoluto"; se formula en las Partidas y fue invocada en diversas ocasiones demandando, más bien, la existencia de un poder personal, no compartido por el monarca con una oligarquía nobiliaria. El pactismo ofreció el argumento a algunos movimientos nobiliarios, pero, también constituyó un argumento de extraordinaria importancia para la legitimación de la dinastía Trastámara, fruto de un contrato tácito entre el pueblo y sus reyes y de la resistencia a los actos tiránicos de un rey convertido, por ello, en ilegítimo.

La monarquía castellana y sus juristas desarrollaron un considerable esfuerzo en la exposición del autoritarismo; sin embargo, ni esta doctrina, ni la opuesta se impusieron de modo completo. El pactismo no pudo hacerlo porque la monarquía castellana - todas las españolas, en general- conservaron considerables resortes de poder en sus manos y pudieron controlar, a veces no sin esfuerzo, a la oligarquía que aspiraba a compartir el poder.

Tampoco el autoritarismo pudo imponerse de modo efectivo. La autoridad del monarca estaba limitada por los privilegios y por las leyes del Reino a cuyo cumplimiento siempre estuvo aquél sujeto. La misma fórmula de "Rey por la gracia de Dios" tantas veces repetida por la cancilleria es un modo de limitación de la autoridad del Rey; en efecto, tal fórmula lo único que realmente dice es que el Rey ha recibido gratuitamente de Dios esa misión. Debe, por tanto, cumplir una serie de obligaciones; en primer lugar las señaladas por la ley divina, de tal manera que ni siquiera podrá legislar ni actuar en contra de lo que podríamos hoy denominar derecho natural.

La autoridad del soberano, por lo tanto, está limitada no sólo por la ley del Reino y los privilegios de sus súbditos, además de por factores de hecho, sino también por las consecuencias de esa actitud ética que 
define el ejercicio de la prerrogativa regia no como un derecho, sino como una obligación que es preciso cumplir.

La legitimidad que ostenta el monarca se transmite por sucesión, principio ya absolutamente establecido desde hacía mucho tiempo; el Rey nace, no se elije. Ello no impide que, en determinadas circunstancias muy especiales, se produzcan conflictos para establecer la sucesión correcta. En estos casos no se impone una solución determinada, sino que, existiendo esa legitimidad, lo único que es preciso hacer es hallar los argumentos que la demuestren con claridad.

En este hecho se basa también la legitimación de los Trastámara. Se llegará a utilizar el derecho sucesorio castellano expuesto en las Partidas, (hay que recordar que fueron norma inspiradora de derecho, pero no Ley del Reino) reconociendo el derecho de representación; de acuerdo con él, Sancho IV no habría sido legítimo rey, sino Fernando de la Cerda y sus sucesores. Dicho de otra manera, la legitimidad habría llegado a Juana Manuel, esposa de Enrique II, lo que legitimaría la revolución Trastámara. Ello no invalida la apelación a otros argumentos como la ilegitimidad de ejercicio en Pedro l, ya comentada, o la búsqueda de otra posible legitimidad, como el matrimonio del futuro Enrique III y $\mathrm{Ca}$ talina de Lancaster, heredera de su abuelo Pedro I.

La institucionalización más visible de la herencia es el otorgamiento de un título especial, Príncipe de Asturias en el Reino de Castilla, que no se adquiere de modo automático, con el nacimiento, sino que debe ser expresamente atribuido. Instituido en 1388 a favor del futuro Enrique III, que lo utilizó lógicamente hasta 1390, no volvió a ser ostentado sino por el futuro Enrique IV, que lo reclamó a su padre en 1444, y vacante de nuevo con la llegada de éste al trono, fue ocupado por Isabel a partir del acuerdo de Guisando.

Al monarca corresponde la totalidad del poder con objeto de regir con justicia el Reino y cumplir la finalidad de sus funciones. Finalidad esencial se considera la defensa del Reino y de los súbditos y la de reinar con justicia, tanto en la vertiente de promulgar leyes justas, como en la de vigilar el cumplimiento de las mismas. El Rey ostenta, por tanto, la suprema autoridad militar, gobierna el Reino y tiene, asimismo, el poder de dictar la ley y el de juzgar su cumplimiento; ostenta pues la plenitud de poderes, es el centro de la administración.

El desarrollo del poder monárquico y la imposibilidad de atender personalmente a todas las funciones propias del gobierno, hizo que se desarrollase considerablemente un aparato burocrático que comparte sus poderes con el Rey. 
El «poder legislativo» era sólo teóricamente atribución exclusiva del Monarca; en realidad este poder viene siendo compartido en cierto modo con las Cortes. En las Partidas se había establecido que el poder de hacer leyes correspondía al Rey y se exigía su aprobación para que cualquier norma elaborada por otros alcanzase rango de ley.

El Ordenamiento de Alcalá (1348) precisa la idea de que la facultad de hacer leyes, modificarlas e interpretarlas corresponde únicamente al monarca. Sólo excepcionalmente, en Olmedo en 1445, en las especialísimas circunstancias del enfrentamiento supremo con los Infantes de Aragón, se llegaría a afirmar que el monarca está por encima de la ley, tomando el argumento del texto de las Partidas. Formulación máxima en un contexto de guerra, no modifica el hecho de que el poder legislativo que corresponde al Monarca, además de obligarle a su cumplimiento, no se suele ejercer sino en colaboración con las Cortes.

Las Cortes de Briviesca de 1387 pidieron que ningún Ordenamiento aprobado en Cortes fuese modificado sino por otro aprobado en igual modo; de hecho se procedió así. Ahora bien, la potestad legislativa no fue monopolizada por las Cortes; los soberanos siguieron dictando leyes sin su concurso. Por ello se introdujo la distinción entre las leyes aprobadas en Cortes, que recibieron el nombre de Ordenamientos, y las publicadas por el monarca, que fueron denominadas Pragmáticas.

La monarquia orienta su esfuerzo no tanto a la publicación en solitario de la Ley, que suscitó la protesta reiterada de las Cortes, como a conseguir la unificación jurídica de todos los territorios del Reino. Es la ya vieja pugna entre el "viejo» y el "nuevo derecho". El viejo, de carácter localista, creaba distintas y aun contradictorias disposiciones sobre idénticos asuntos; el derecho común fue impulsado por los monarcas y en esa línea se inscriben la redacción de las Partidas en época de Fernando IV, la tercera de este texto jurídico, y el Ordenamiento de Alcalá realizado por Alfonso XI. Con él se imponía una uniformidad legislativa que establecía como primera ley el propio Ordenamiento, en segundo lugar los fueros municipales y, en fin, las Partidas. No obstante prosiguió la vigencia de numerosas normas consuetudinarias, especialmente en algunas regiones del Reino.

El «poder judicial» no solamente es detentado por el Monarca, sino que se considera que, precisamente, la administración de justicia es la función esencial de la Monarquía. Ésta es ejercida por el monarca, en su nombre por los jueces ordinarios y, por delegación suya, los "señores" en sus "señoríos". 
El Rey es, en fin, la cabeza visible del Reino, rige sus destinos, dirige las relaciones exteriores, declara la guerra y concierta la paz, convoca y manda el ejército, rige la administración, y administra sus recursos; es decir, ostenta también el «poder ejecutivo».

\section{LA ADMINISTRACIÓN CENTRAL}

El monarca ostenta la plenitud de todos los poderes, pero no los ejerce en solitario; es auxiliado en sus funciones por personas de su confianza, a quienes tiene cerca de sí, y, a medida que la complejidad administrativa es mayor, por organismos de gobierno poblados de una burocracia creciente.

El origen de la administración es el conjunto de personas, laicos y eclesiásticos que acompañan habitualmente al monarca a quien éste acostumbra a someter la adopción de decisiones. Este conjunto de personas es designado con el nombre de "Curia" o "Corte».

Habitualmente, sólo el reducido número de personas que integran la "Curia" toma parte en las decisiones, pero, extraordinariamente, con ocasión de decisiones de especial importancia o en momentos graves para la vida del Reino, suelen los monarcas convocar a un mayor número de personas con las que estudian las decisiones a adoptar, o que aprovechan para dar solemnidad a ciertas disposiciones. La primera suele recibir el nombre de "Curia ordinaria», reservándose a la segunda la denominación de "Curia extraordinaria» o «plena».

En cualquiera de sus versiones la "Curia" es un consejo y alto tribunal al que el monarca somete los más variados asuntos; en ningún caso sus opiniones son vinculantes aunque es evidente que sus opiniones hubieron de ser tenidas muy en cuenta.

De la Curia van a nacer instituciones diferentes de extraordinaria importancia para la vida del reino. De las reuniones de la "Curia ordinaria» nace el "Consejo»; de las reuniones de la "Curia plena" nacerán las Cortes que tienen su configuración definitiva a partir del momento en que a sus sesiones se incorporan los representantes de las ciudades del Reino. La mayor complejidad de las funciones de gobierno irá haciendo nacer nuevos organismos, desgajados de la Corte, que irán diferenciándose asímismo entre los que tienen carácter esencialmente doméstico, de los de carácter público. 


\section{La Casa del Rey}

Abarca a todos los que se ocupan de las necesidades de gobierno y administración de la misma; en teoría con carácter doméstico, no siempre es fácil distinguirlo ya que sus funciones se extienden a la administración del Reino en general.

El primer oficial es el Mayordomo, encargado de la dirección de los servicios del Palacio y de la administración de la Corte. La complejidad de los servicios de la Corte aconsejaría la existencia de diversos mayordomos a los que se situó bajo la autoridad de un Mayordomo Mayor.

A las órdenes del Mayordomo se hallan todos los oficiales de carácter doméstico, aunque sus funciones se extienden a lo público. El Mayordomo había sido definido en las Partidas como aquel a quien correspondía el mantenimiento de la casa del Rey, toma cuenta de los gastos y controla las cuentas de los demás oficiales. En la época ahora estudiada existían ya varios mayordomos, a la cabeza de los cuales se sitúa el llamado Mayor; su importancia se deduce fácilmente por el lugar destacado que ocupa en la confirmación de privilegios, al lado del alférez, jefe supremo del ejército.

Son funcionarios directamente sometidos al Mayordomo el camarero, tesorero, almojarife y contadores. El camarero es el custodio de la cámara regia y de sus bienes, ropas y alhajas; su función es evidentemente doméstica, pero sobrepasa ese cometido para convertirse en una función política. Es uno de los primeros cargos en convertirse en hereditario, y, precisamente sobre él, edifican los Velasco su fuerza política.

El tesorero confunde a veces sus funciones con las del camarero, pero va especializándose en el control de cuentas, auxiliado por contadores hasta dar lugar a un organismo especializado, organizado en época de Juan II y asentado en Valladolid. Como institución hacendística ha de tratarse en otro lugar.

Existen también otros funcionarios domésticos como despenseros, reposteros, aposentadores, fisicos, porteros, halconeros, monteros y caballerizos, etc., cuya definición no parece necesaria.

Muchos de los oficios domésticos se convirtieron en honorificos siendo desempeñados por miembros de la alta aristocracia, desposeídos de su contenido inicial puramente doméstico $y$, a veces bastante humilde. Se da también un esfuerzo de distinción de funciones y de su marco que, en el siglo $\mathrm{XV}$, se manifiesta en las retribuciones que cada uno percibe: los emolumentos de los que tienen funciones de carácter público se de- 
nominan "quitaciones»; los de quienes están al servicio de la casa del Rey cobran «raciones».

\section{La Cancillería}

En el camino de la especialización de funciones que sigue la administración central, el primer organismo que ha conocido vida independiente había sido la Cancillería. Organizada durante el reinado del Alfonso VIl, se le confía la redacción y expedición de documentos; a las órdenes del canciller existe un adecuado personal de notarios, secretarios, etc.

El canciller se convierte pronto en un alto oficial de la corte, incluso se vincula el cargo a determinados eclesiásticos, el arzobispo de Santiago, en el caso del reino de León, y el de Toledo en el de Castilla. Como en otros casos, la conversión en honorífico del cargo llevaba consigo la existencia de un "Canciller Mayor» en el reino de León y otro en el de Castilla, vinculados a quien se ha dicho, y que el desempeño efectivo del cometido se encargase a otros cancilleres, normalmente del Rey, de la Reina y de la poridat, que actúa en asuntos reservados.

También la Cancillería tiende a fijarse en un lugar determinado desde comienzos del siglo XV. En las Cortes de Madrid de 1419 se decide su establecimiento en Segovia, aunque a finales del reinado de Juan II se instala en Valladolid.

Dentro de la Cancillería destacan como responsables de su funcionamiento diversos notarios mayores a los que se atribuyen circunscripciones. Vienen funcionando, ya desde el siglo XIII los de León, Castilla y Andalucía a los que se une, desde comienzos del siglo XIv, una nueva circunscripción: Toledo.

\section{Audiencia y Chancillería}

La función judicial es, como se ha dicho, una de las funciones más importantes de la monarquía. El monarca tiene en esta función una misión esencial; a él le corresponde y todos los jueces actúan por su delegación y como representantes suyos. Las reuniones de la Curia, como ya se ha dicho, tenían una función judicial muy definida. 
Naturalmente existen numerosos tribunales que ejercen esta función en representación o por delegación del rey: asambleas judiciales de distrito, tribunales municipales, condales o señoriales.

Durante mucho tiempo la administración de justicia no se distingue de modo pleno de otras actividades de gobierno. Lo será sobre todo a partir del siglo XIII, a través del mismo proceso que está produciendo la especialización de funciones administrativas y también como efecto de la recepción del derecho romano y los innovaciones de carácter procesal.

Las funciones judiciales del monarca no solamente no se diluyen, sino que se intensifican. Se organiza, desde el reinado de Alfonso X, dentro de la Curia, un «tribunal de la Corte», presidido teóricamente por el Rey. No obstante, su presidencia habitual corresponde a un «sobrejuez" o "adelantado mayor de la Corte».

En 1274, en las Cortes de Zamora, se organiza un tribunal que actuaría en la Corte pero con independencia de la Curia. De este tribunal, cuyas sesiones tendrian lugar lunes, miércoles y viernes, forman parte, además del Rey, el «sobrejuez» y veintitrés jueces o «alcaldes de Corte» - nueve para Castilla, ocho para León y seis para Extremadura-que deberán acompañar siempre al Rey para aplicar la justicia allí donde éste se encuentre. Su misión es ocuparse de los llamados "casos de Corte", es decir, de los delitos de especial gravedad, que se definen en el propio ordenamiento. También forman parte de este tribunal tres «alcaldes de las alzadas", peritos en derecho que entienden las apelaciones sobre las sentencias emitidas por otros jueces.

La constitución de un tribunal regio constituía, de hecho, una consolidación del poder de la monarquía; la no inclusión entre sus componentes de miembros de la nobleza constituyó, además, otro motivo de queja que dificultó notablemente la aplicación del Ordenamiento de las Cortes de Zamora.

Se reiteró, también con escaso éxito, esta disposición en las Cortes de Valladolid de 1299 y se procedió a una completa reestructuración, nuevamente en Valladolid, en 1312. El tribunal de la Corte queda constituido por doce jueces, hombres buenos, la mitad de los cuales debería acompañar siempre a la Corte, actuando durante medio año. Las Cortes de Madrid de 1329 dispusieron, finalmente, que entre los alcaldes de Corte hubieses necesariamente alguno de condición noble.

Como en otros aspectos, la estructuración más importante se lleva a cabo bajo los Trastámara. Apenas triunfante Enrique II, en las Cortes de Toro de 1371, se crea una «Audiencia» como tribunal permanente de la 
Corte; lo integran siete «oidores», tres prelados y cuatro jurisperitos, que tendrían sesión tres veces por semana en la corte, en la residencia del Canciller o donde se hallase la cancillería o chancillería, nombre que acabaría designando también a la Audiencia.

En cuanto a los alcaldes de corte, el ordenamiento establecía que fuesen ocho - dos por Castilla, dos por León, dos por Extremadura, uno por Toledo y uno por Andalucía- que intervendrían en los casos de corte de su correspondiente jurisdicción; habría también dos «alcaldes del Rastro" - territorio en torno a la residencia regia- que actuarian en su función concreta y, también, junto con los alcaldes ordinarios, en los pleitos criminales, y un "alcalde de las alzadas» con competencia en las apelaciones.

El ordenamiento instituia también un alcalde de los «fijosdalgo» encargado de juzgar las causas y litigios de los nobles. Era una modificación sustancial destinada a suprimir la resistencia nobiliaria a la creación de la Audiencia. Teniendo en cuenta que la monarquía Trastámara - por más justificaciones de carácter jurídico que quieran aducirse- era el resultado de una asonada nobiliaria, no tiene nada de extraña esta modificación.

A pesar del ordenamiento, el funcionamiento de la justicia parece poco satisfactorio, escasamente flexible ante las apelaciones, lento, ventajoso para quienes están próximos al Rey y complejo por la frecuente superposición de jurisdicciones. Este último hecho es especialmente apreciable respecto a la jurisdicción eclesiástica y a la de la Mesta, cuyos jueces invadian la esfera de la jurisdicción ordinaria, suscitando reiteradas protestas en las Cortes.

El eco de tales protestas es especialmente apreciable, aunque todas las sesiones de Cortes se ocupan de la justicia, en las de Burgos de 1379 y en las de Segovia de 1386, en las que Juan I prometió una reforma a fondo que, sin embargo, no pudo llevar a efecto. El poder eclesiástico y los grandes intereses económicos que controlaba la Mesta hacian sumamente difícil atender las reclamaciones presentadas.

Las medidas más importantes referidas a la Justicia se adoptaron en 1383 y 1387 . En las Cortes de Segovia de 1383 el monarca prometía ocuparse personalmente de la justicia y se sentaría en la Audiencia todos los viernes - como un emir musulmán, apunta Luis Suárez- medida arcaizante, sin duda, que, además, no pudo realizarse. No obstante, se adoptaron otras medidas de interés, especialmente la obligación de oidores y alcaldes de residir en la Corte y de asistir a las sesiones de la Audiencia. 
La reorganización más profunda de la Audiencia fue abordada en las Cortes de Briviesca, de 1387, como también del Consejo, al que nos referiremos más adelante. En esta ocasión se pretendió independizar totalmente la administración de Justicia de los otros servicios administrativos y convertir a la Audiencia en el tribunal de apelación por excelencia, limitando drásticamente los casos de apelación al Rey, sólo posible en circunstancias excepcionales.

Se aumentaba el número de oidores a ocho, que actuarían por mitades, como los alcaldes de Corte; se les asignaban «quitaciones» dobles durante los meses de actuación y, lo más importante, se fijaba la residencia de la Audiencia en cuatro lugares, permaneciendo tres meses en cada uno de ellos: Medina del Campo - abril a junio-, Olmedo - julio a septiembre-, Madrid -octubre a diciembre-, y Alcalá de Henares -enero a marzo-. Era una solución intermedia, y también arcaizante, porque, aunque la Audiencia dejaba de ser ambulante, no tenía una residencia fija.

Una reforma de carácter procesal -dando mayor importancia al procedimiento escrito- fue introducida en el funcionamiento de la Audiencia en las Cortes de Guadalajara de 1390. En estas mismas Cortes se estudiaron los graves inconvenientes que para el funcionamiento de la Audiencia tenía el traslado de residencia. Se fijó ésta en Segovia y se modificó nuevamente el número de miembros de la Audiencia, compuesta, desde ahora, por 16 Oidores - seis prelados y 10 letrados- dos alcaldes de los fijosidalgos, uno de las "alzadas" y los ocho alcaldes de Corte.

Durante el reinado de Enrique III se suprime la Audiencia, siendo impartida la Justicia por un único oidor en representación del Rey; esta modificación significaba una vuelta atrás, un teórico ejercicio de la Justicia por el monarca, que ya no podía tener viabilidad. En 1407, fallecido Enrique III, se restableció la Audiencia tal como había quedado diseñada en época de Juan I.

Concluida la minoria de Juan II, en las Cortes de Madrid de 1419, se disminuyó el número de oidores a 10 -ocho letrados y dos prelados- y se insistió en fijar la Audiencia en Segovia; sin embargo, de hecho, funcionó en Valladolid, sin duda en razón de la Universidad vallisoletana. En las Cortes de Palenzuela de 1425 se disminuyó nuevamente el número de oidores a siete y se reguló la residencia por medio año alternativamente, en Turégano y Griñón, volviendo al mismo sistema previsto en 1387 y que había demostrado su inviabilidad. 
En realidad, no se produjo desplazamiento de la Audiencia que, la mayor parte del tiempo, siguió funcionando en Valladolid; de hecho, en las cortes celebradas en esta ciudad en 1442, se ordenó que ésta fuese la sede de la Audiencia y en ella permaneció en el futuro, aunque no de modo totalmente continuado, hasta su definitiva fijación en la ciudad castellana por los Reyes Católicos. Mientras tanto, durante el reinado de Enrique IV, y, especiaimente, durante la guerra civil, la Audiencia sufrió importantes alteraciones tanto en su funcionamiento como en el número de sus miembros.

La reorganización del Estado acometida por los Reyes Católicos se ocupó también de la reordenación de la Audiencia, una de las instituciones que había sufrido más trastornos en época de su predecesor. La reforma fue estudiada en las Cortes de Toledo de 1480, encargándose cuatro años después al arzobispo de Compostela, Alfonso de Fonseca, nombrado presidente de la Audiencia, preparar un estudio sobre el ordenamiento definitivo de la institución.

Sus propuestas son las que recoge el Ordenamiento publicado en 1489. Se pretendía una total independencia de la Audiencia y Chancillería respecto a los demas organismos del gobierno central, se fijaba su residencia en Valladolid y se disponía su organigrama interno. En el futuro estaría compuesta por un obispo, que actuaría como presidente, ocho oidores, varios alcaldes, dos procuradores fiscales, dos abogados de pobres, escribanos y relatores. Se dividía en tres secciones, dos de las cuales entendían en asuntos civiles - casos de corte, suplicaciones y apelaciones - y otra se ocupaba de lo criminal. Después surgiría la sección de hidalgos y la de Vizcaya.

También se regularon numerosas cuestiones referentes al procedimiento, actuación de los oidores, sueldos, sentencias, así como los requisitos que debían cumplirse en interrogatorios, aplicación de penas, incompatibilidades, etc.

A pesar del esfuerzo de definición de organización y funciones, existían motivos de confusión. Resulta especialmente difícil definir las respectivas competencias de la Audiencia, del Consejo Real y de los alcaldes de urastro", todos ellos con competencia en asuntos civiles y criminales.

Al fijarse la residencia en Valladolid, la Audiencia resultaba excesivamente excéntrica para algunas regiones, por lo que se creó, en 1494, una nueva Audiencia y Chancillería en Ciudad Real que, en 1505, sería trasladada a Granada. Sería, con la de Valladolid, la única denominada Chancillería, ostentando todas las demas que se creen, únicamente, el nombre de Audiencia. Es el caso de la de Compostela, donde, desde 
1480 , viene funcionando una audiencia de Justicia, integrada por cuatro oidores, aunque no se instaure como verdadera Audiencia hasta 1505.

\section{El Consejo Real}

El cuerpo esencial de la administración centrai lo constituye, en el siglo XV, el Consejo Real, mezcla de funciones propias del poder ejecutivo y de otras de carácter judicial, hasta el punto de poderse hablar de una verdadera yuxtaposición de órganos de gobierno y justicia.

De modo unánime, se considera que los orígenes del Consejo se hallan en la Curia ordinaria de la que forman parte la familia real, los oficiales más importantes y los magnates laicos y eclesiásticos que de modo permanente $u$ ocasional acompañan al monarca. No se trata de un organismo con personalidad y funciones definidas, sino de un conjunto de personas que, de modo absolutamente normal, cumplen el deber feudal del "consilium»; no se precisa una gran explicación de carácter institucional: es lógico que el monarca guste, espontáneamente, de someter decisiones de importancia a la opinión de quienes gozan de su confianza y amistad.

Después, del mismo modo que se van configurando otros organismos administrativos, el Consejo irá adquiriendo una mayor personalidad y definición. La complejidad administrativa y la difusión de una normativa jurídica, irán exigiendo, como en el resto de la actividad administrativa, la participación de juristas.

Es posible que fuese en época de Fernando III cuando adquiriese una cierta configuración, o se incorporasen, de modo habitual, a las tareas de consejo hombres de derecho. En lo sucesivo, aunque ninguna noticia concreta habla de la existencia de un «Consejo Real», son abundantes las referencias al hecho de que el Rey somete tal o cual cuestión al consejo de ciertas personas, a los consejeros del Rey, etc.

Durante los siglos XIII y XIV, si no existió un organismo definido, que pueda ser señalado como el consejo real, sí puede afirmarse que, habitualmente, los monarcas cuentan con el asesoramiento de un determinado número de personas; especialmente durante las minorías, este consejo tuvo una notable importancia y de él formaron parte normalmente, sobre todo cuando actúa como tribunal, un cuerpo de juristas.

$\mathrm{Ni}$ se halla estrictamente reglamentado el número y funciones de sus miembros, periodicidad de reuniones, ni, mucho menos aún, sus delibe- 
raciones tuvieron carácter vinculante para el soberano. Sin embargo, cuando se regula la existencia y actuación del consejo, no es algo plenamente nuevo, sino la reglamentación de algo que, desde hace tiempo, viene funcionando de forma habitual.

La institucionalización del Consejo Real tiene lugar en el reinado de Juan I, como un exponente más de la reordenación acometida durante el reinado de este monarca. El Consejo Real fue creado en las Cortes de Valladolid de 1385 y en la exposición de motivos se apela tanto a los precedentes de su funcionamiento, como a la petición expresada repetidamente por los procuradores, incluso a la costumbre de otros reinos, clara referencia a Aragón y al Ordenamiento de Pedro IV.

El Consejo se organizaba como un órgano autónomo, no representativo; aunque sus miembros procediesen de los diversos estamentos que integran las Cortes, no era el Consejo una permanente de aquéllas; no obstante fue inevitable que se diese esa sensación y contra ella se luchó desde el primer momento.

El Consejo estaría integrado por 12 consejeros, cuatro obispos -Toledo, Compostela, Sevilla y Burgos-, cuatro caballeros -el marqués de Villena, Juan Hurtado de Mendoza, Pedro Suárez de Quiñones y Alfonso Fernández de Montemayor-, y cuatro ciudadanos: Juan de Sanjuanes, Ruy Pérez de Esquivel, Ruy González de Salamanca y Pedro García de Peñaranda.

La designación no obedecía a ningún criterio establecido, sino a la voluntad del Rey; ni ocupaban su puesto, como hemos dicho, en representación de nadie; sin embargo, resulta evidente que se producía una verdadera delegación de funciones, una autoridad, o, al menos, una responsabilidad compartida. El Consejo actúa con plena autonomía y sus decisiones, tomadas en nombre del Rey, no precisan siquiera la firma del monarca. Éste se reserva el nombramiento de oficios de Casa y Audiencia, las presentaciones para las Iglesias, la concesión de gracias y mercedes y el indulto de homicidios, si bien manifiesta su deseo de actuar en estas cuestiones contando con la opinión del Consejo.

En las Cortes de Briviesca de 1387 se volvió a reorganizar el Consejo. La modificación más importante fue, sin duda, la sustitución de los procuradores de las Cortes por doctores en Derecho. Conviene recordar que son los juristas quienes defienden las doctrinas que sustentan la autoridad monárquica; pero, sobre todo, se aprecia el deseo de impedir que el Consejo pudiese parecer una permanente de las Cortes. Por supuesto, el Monarca reiteraba toda negativa a aceptar cualquier limitación en la designación de miembros del Consejo. 
Se regulaba también el funcionamiento del Consejo: reunión diaria, en sesiones de mañana y tarde, secreto de las deliberaciones del mismo, existencia de libro de actas, etc. Dos años después, en 1389, Juan I dispuso que el Consejo despachase necesariamente con él en una de las dos sesiones del día; en 1390 se dictaban las últimas normas respecto al funcionamiento del Consejo, estableciéndose el procedimiento de actuación, el orden de intervención de los Consejeros, la duración de las sesiones, la hora de comienzo de las mismas, etc. Quedaba configurado como un auxiliar imprescindible del monarca en la labor de gobierno.

La minoría de Enrique III provocó una fusión del Consejo Real y del de Regencia y, nuevamente, la incorporación de procuradores de las Cortes. En 1406 este Monarca reorganizó el Consejo en el mismo sentido que había dictado su padre, es decir, eliminando a los procuradores, sustituidos por juristas, y estableciendo el número de consejeros en dieciséis.

La minoría de Juan II, más larga que la de su padre, planteó al funcionamiento del Consejo los mismos problemas que en aquélla. La pugna entre los Infantes y don Álvaro tendría consecuencias decisivas sobre el Consejo, convertido en instrumento en la lucha entre unos y otros; su control constituyó el objeto mismo de la lucha, y, del análisis de los miembros que lo integran en uno u otro momento, se deduce quien es el dueño de la situación. Naturalmente el número de consejeros creció de modo importante y su funcionamiento como institución de gobierno se resintió considerablemente.

La sustitución de miembros del Consejo y la toma por sí mismo de su presidencia fueron los primeros actos realizados por el infante don Enrique, en julio de 1420, en lo que conocemos como golpe de Estado de Tordesillas. Pocos meses después, don Álvaro desplaza del gobierno a don Enrique y procede a una nueva modificación de la composición del Consejo, repetida sistemáticamente a cada cambio de situación: tras el pacto de Torre de Arciel de septiembre de 1425; después del destierro de don Álvaro, en agosto de 1427; a su vuelta a la Corte, pocos meses después; en 1439, con el nuevo destierro de don Álvaro; a su nuevo regreso, y así sucesivamente.

Tal situación hacía inviable el funcionamiento del Consejo. Por eso las Cortes de Valladolid de 1440 solicitaron de Juan II la aplicación de la ordenanza de 1406, que, efectivamente fue reiterada en 1442. Sin embargo no era posible un retorno simple al pasado: el Consejo se había convertido en un organismo esencial del grupo nobiliario. En la práctica hubo dos Consejos: el del Rey y los nobles, en el que se adoptaban las 
decisiones políticas, y un Consejo, integrado por seis caballeros, cuatro doctores en derecho y dos prelados, con funciones judiciales exclusivamente.

Aunque con el mismo nombre, resulta evidente que este Consejo tiene poco que ver con el diseñado por Juan I. Esta situación se mantiene durante gran parte del reinado de Enrique IV: ni existe número fijo de consejeros ni su composición es otra cosa que el indicativo del grupo que controla el poder.

En 1459 una ordenanza disponía que sus integrantes fueran ocho juristas, dos caballeros y dos prelados, lo que reafirmaba su carácter judicial, referido naturalmente a ese Consejo judicial, no al que podriamos denominar ejecutivo. Además las tensiones que vive el Reino a partir de esta fecha hicieron que fuera nula la aplicación de esta ordenanza.

Como tantas otras cuestiones e instituciones, se requería en el Consejo una eficaz reorganización en el momento de acceder al trono los Reyes Católicos. De esa tarea general de reorganización - económica y administrativa - se ocupan especialmente las Cortes de Toledo de 1480.

De acuerdo con las deliberaciones de esas Cortes, los Reyes Católicos decretaron una Ordenanza que remodelaba absolutamente el Consejo. Se establecía una diferencia esencial entre los consejeros permanentes, ocho juristas, tres caballeros y un prelado, y los numerosos prelados, magnates y Maestres de Órdenes Militares, también denominados consejeros, aunque su participación fuese más o menos ocasional. Aunque éstos últimos eran más importantes personalmente, resulta evidente la creciente importancia de los permanentes. Hay también un desarrollo de la burocracia en la que descuellan algunas personas cuya proximidad a los Soberanos le confiere una excepcional importancia.

El Consejo se divide en cinco secciones que actúan en salas diferentes del Palacio; la primera, se encarga de las relaciones internacionales; la segunda, entiende en las apelaciones de justicia; la tercera, de Hacienda, se encarga de la administración financiera, junto con los Contadores y agentes fiscales; la cuarta, reunía a los representantes de la Hermandad y la quinta entendía en los asuntos de Aragón, hasta que, en 1494, se creó el Consejo privativo de aquel Reino.

La autonomía de funcionamiento de los Consejos era muy considerable; así, junto a su decisiva importancia en los asuntos «librados por Cámara", es decir, estudiados conjuntamente con los Reyes, numerosas cuestiones eran decididas por la propia autoridad del Consejo, «libra- 
miento por expediente", lo que le convierte, de hecho, en la máxima autoridad, siempre, desde luego, en perfecta sintonía con los Monarcas.

La especialización funcional del Consejo le convierte en algo más que un ente consultivo; es el punto de partida de una administración central moderna que atribuye a estos órganos las funciones no sólo ejecutivas y judiciales, sino, en muchos casos, legislativas. 